\title{
Antitumor immunity targeting fibroblast activation protein- $\alpha$ in a mouse Lewis lung carcinoma model
}

\author{
JUNPING XIE, SHIYANG YUAN, LAISHUI PENG, HUANYU LI, LINXIA NIU, \\ HUI XU, XIAOLIN GUO, MEI YANG and FENGYING DUAN
}

\begin{abstract}
Department of Respiratory and Critical Care Medicine, The Second Affiliated Hospital of Nanchang University, Nanchang, Jiangxi 330006, P.R. China
\end{abstract}

Received September 12, 2019; Accepted April 15, 2020

DOI: $10.3892 / \mathrm{ol} .2020 .11637$

\begin{abstract}
The tumor stromal microenvironment is an integral part of the occurrence and development of tumor. Cancer-associated fibroblasts (CAFs) are a key component of most tumor stromal microenvironments. The present study aimed to investigate the use of CAFs-targeted immunotherapy to fibroblast activation protein- $\alpha$ (FAP- $\alpha$ ) expressed in CAFs. Recombinant adenoviral vectors containing the mouse FAP- $\alpha$ cDNA (rAd-FAP- $\alpha$ ) were constructed. C57BL/6 mice were immunized with rAd-FAP- $\alpha$ infected dendritic cells (DCs) against FAP- $\alpha$, which is overexpress in CAFs. The results demonstrated that mice vaccinated with rAd-FAP- $\alpha$ DCs gave rise to potent FAP- $\alpha$-specific cytotoxic T lymphocytes capable of lysing Lewis lung cancer (LLC) CAFs. Furthermore, mice vaccinated with rAd-FAP- $\alpha$-transduced DCs induced an effective therapeutic or protective antitumor immunity to LLC in a subcutaneous model, and prolonged overall survival time compared with mice vaccinated with the control recombinant adenovirus-transduced DCs (rAd-c DCs) or DCs alone. The results of the present study suggested that FAP- $\alpha$, which is preferentially expressed in CAFs, may be considered as a potential target for killing or destroying CAFs within the tumor stromal microenvironment, and may be exploited to develop immunogenic tumor vaccines.
\end{abstract}

\section{Introduction}

Tumors consist of different populations of cells, including both transformed and untransformed cells (1). The untransformed cells include infiltrated inflammatory cells, endothelial cells, immunocytes, interstitial-derived smooth muscle cells,

Correspondence to: Dr Junping Xie, Department of Respiratory and Critical Care Medicine, The Second Affiliated Hospital of Nanchang University, 1 Minde Road, Nanchang, Jiangxi 330006, P.R. China

E-mail: junpingxie@sina.com

Key words: antitumor immunity, cancer-associated fibroblast, fibroblast activation protein $\alpha$, Lewis lung cancer, dendritic cells cancer-associated fibroblasts (CAFs) and pericytes, which are herein collectively referred to as interstitial cells $(1,2)$. Interstitial cells communicate among themselves and with tumor cells, immunocytes and inflammatory cells directly through cell contact or indirectly through exocrine/paracrine effects, the use of proteases and regulation of the extracellular matrix $(3,4)$. This complicated communication network serves a key role in providing an appropriate microenvironment to sustain tumor occurrence, angiogenesis and metastasis $(3,4)$. Because of the pivotal role of the tumor stromal microenvironment in tumorigenesis and development, identification of stromal targets for cancer therapy is critical and may provide improved strategies to directly target tumor cells. In cancer stromal cells, CAFs may be required for tumor cells to proliferate and survive in vivo (5-8). CAFs represent a heterogeneous cell population, and their phenotype may be different from that of normal fibroblasts, such as secreting different cytokines or expressing different proteins $(9,10)$. Previous studies have indicated that fibroblasts regulate the proliferation of cancer cells that may appear as normal cells in the early stages of tumorigenesis $(11,12)$. Although the functional and phenotypical heterogeneity of CAFs remain unclear, CAFs have been characterized as myofibroblasts, in part according to $\alpha$-smooth muscle actin ( $\alpha$-SMA) expression (13).

Fibroblast activation protein- $\alpha$ (FAP- $\alpha)$, also known as FAP or seprase, has been identified as a marker of reactive fibroblasts in cancer (including CAFs), fibrotic lesions and granulation tissue (14-16). FAP- $\alpha$ has attracted interest through its potential role as a therapeutic target due to its regulated expression in the stroma of cancerous lesions and the structural evidence of its proteolytic activity (14-18). However, its function in cancer remains largely unclear. FAP- $\alpha$ is the overexpression product of CAFs and is the predominant component of the tumor stroma (19). CAFs are different from adult normal tissue fibroblasts and instead resemble wound healing-associated and early human fetal fibroblasts (19). CAFs are key regulators of tumorigenesis; however, they are more genetically stable than cancer cells (13). CAFs may therefore represent more feasible therapeutic targets for tumor immunotherapy compared with cancer cells (13).

In order to specifically target CAFs and investigate the immunogenicity of the FAP- $\alpha$ protein, the present study used an immunity method, applying $\mathrm{H}-2^{\mathrm{b}}$ positive murine bone 
marrow-derived dendritic cells (DCs) transfected with a recombinant adenovirus carrying the FAP- $\alpha$ gene (rAd-FAP- $\alpha$ ), in order to induce the antitumor immune response against subcutaneous implanted Lewis lung carcinoma (LLC) in C57BL/6 mice.

\section{Materials and methods}

Cell line and mice. LLC cells $\left(\mathrm{H}-2^{\mathrm{b}}\right)$ were provided by The Cell Bank of Type Culture Collection Academy of Sciences, whereas 293T cells were purchased from the American Type Culture Collection. Cells were cultured in DMEM medium supplemented with $10 \%$ fetal bovine serum (FBS; Biological Industries) at $37^{\circ} \mathrm{C}$ in a humidified incubator containing $5 \%$ $\mathrm{CO}_{2}$

A total of 70 female C57BL/6 $\left(\mathrm{H}-2^{\text {b }}\right)$ mice (age, 7-8 weeks; weight, 18-24 g) were purchased from the Laboratory Animal Research Institute at Tongji Medical College of Huazhong University of Science and Technology (Wuhan, China). All mice were kept in individual ventilated cages with food and water ad libitum, at controlled temperature conditions $\left(22 \pm 1^{\circ} \mathrm{C}\right)$, with a $12 \mathrm{~h} \mathrm{light/dark}$ cycle at $50 \%$ humidity. All experimental protocols were approved by the Ethics Committee of The Second Affiliated Hospital of Nanchang University (Nanchang, China; approval no. NDEFYEC 175-2018) and mice were sacrificed according to the Institutional Animal Care and Use Committee protocol.

Construction of mouse FAP- $\alpha$ adenovirus vector. A cDNA-encoding mouse FAP- $\alpha$ of CAFs was purchased from Open Biosystems Inc. The FAP- $\alpha$ fragment was amplified with specific primers. The sense PCR primer, including a BamHI site (underlined) was 5'-AGGTCGACTCTAGAGGATCCG CCACCATGAAGACATGGCTGAAAACTGTC-3', whereas the anti-sense primer, including an AgeI site (underlined) was 5'-TCACCATGGTGGCGACCGGTCAGTCTGATA AAGAAAAGCATTGC-3'. The amplified cDNA was digested with BamHI and AgeI, and inserted into the GV137 plasmid (Shanghai GeneChem Co., Ltd.) to yield GV137-FAP- $\alpha$. PCR was subsequently performed to verify the successful construction of this shuttle plasmid. FAP- $\alpha$ cDNA was amplified in a $50 \mu \mathrm{l}$ reaction containing: $5 \mu \mathrm{l} 10 \mathrm{X}$ AmpliTaq Gold Polymerase buffer, $5 \mu \mathrm{l}$ dNTP $(25 \mathrm{mM}$ ), 20 pmol (final concentration) specific forward/reverse primers and $1 \mu 1$ AmpliTaq Gold Polymerase (Thermo Fisher Scientific, Inc.). The thermocycling conditions used for PCR were as follows: Initial denaturation at $95^{\circ} \mathrm{C}$ for $10 \mathrm{~min} ; 25$ cycles at $95^{\circ} \mathrm{C}$ for $30 \mathrm{sec}, 55^{\circ} \mathrm{C}$ for $30 \mathrm{sec}, 72^{\circ} \mathrm{C}$ for $30 \mathrm{sec}$; and a final extension at $72^{\circ} \mathrm{C}$ for $7 \mathrm{~min}$. The primer sequences used for PCR were as follows: FAP- $\alpha$ forward, 5'-TTCTGCCTCCTCAGTTTG-3' and reverse, 5'-CTGGTCGAGCTGGACGGCGACG-3'. The expected size of the fragments was $795 \mathrm{bp}$. Following co-transfection with 4 ug the helper plasmid [pBHGlox $(\delta)$ E1,3Cre] and 2 ug the shuttle plasmid (GV137-FAP- $\alpha$ ) (both from Hanheng Biotechnology Shanghai Co., Ltd; https://www. hanbio.net) into 293T cells, the recombinant adenovirus vector containing the mouse FAP- $\alpha$ cDNA (rAd-FAP- $\alpha$ ) was generated using the AdMax kit D (Microbix Biosystems, Inc.). After 12 days when typical cytopathic effects (CPE; CPE is defined as degenerative changes in cell morphology associated with the replication of adenoviruses. These morphologic changes, such as cell rounding and lose of cell adhesion, can be detected via fluorescence microscopy) $(20,21)$ were exhibited for all cells and $>50 \%$ of cells were detached from the flask, the transfected cells were collected. Subsequently, the transfected cells were washed once with PBS, frozen and thawed three times at $-70 / 37^{\circ} \mathrm{C}$, respectively. The cell lysate was centrifuged at $1,000 \times \mathrm{g}$ for $10 \mathrm{~min}$ at $4^{\circ} \mathrm{C}$ and the supernatant was collected. The initial supernatant was the crude lysate that included rAd-FAP- $\alpha$, which was expanded in vitro, and purified via density gradient centrifugation and dialysis. Briefly, the fast sealed tube containing the prepared iodixanol gradient (cat. no. D1556-250; Sigma-Aldrich; Merck KGaA) and the lysate was centrifuged at $500,000 \times \mathrm{g}$ for $90 \mathrm{~min}$ at $18^{\circ} \mathrm{C}$, using a rotor $70 \mathrm{Ti}$ (Beckman Coulter, Inc.). In order to retrieve the virus, the tube was fixed on a holder set at eye level and the $18 \mathrm{G}$ needle was subsequently inserted into the top of the tube to allow air to enter. Another $18 \mathrm{G}$ needle was attached to a $10 \mathrm{ml}$ syringe and inserted directly below the interface of the 60 and $40 \%$ iodixanol layers, with the slope of the needle facing upwards. $\sim 4 \mathrm{ml}$ of sample containing the recombinant adenovirus was extracted from the $40 \%$ iodixanol layer $(20,21)$. The rAd-FAP- $\alpha$ titers were determined using an endpoint dilution assay, as previously described (20,21). A recombinant adenovirus containing an enhanced green fluorescent protein (GFP) gene was generated using a similar method used to construct the control adenovirus (rAd-c) [Hanheng Biotechnology Shanghai Co., Ltd $(20,21)]$.

Generation of DCs and transduction with rAd-FAP- $\alpha$. Bone marrow (BM)-derived DCs were produced from mouse myeloid progenitor cells as previously described (21). Briefly, two $\mathrm{C} 57 \mathrm{BL} / 6$ mice were sacrificed by inhaling $\mathrm{CO}_{2}\left(\mathrm{CO}_{2}\right.$ chamber-replacement rate of $30 \%$ for $15 \mathrm{~min}$ ). After removing all muscle tissues from the mice tibias and femurs, BM progenitors were flushed out. The red blood cells (RBC) were lysed with $10 \mathrm{mM}$ ammonium chloride Tris buffer (Sigma-Aldrich; Merck $\mathrm{KGaA}$ ) at room temperature for $2 \mathrm{~min}$. Cells were subsequently centrifuged at $300 \mathrm{x} \mathrm{g}$ for $5 \mathrm{~min}$ at room temperature and washed twice with DMEM. The remaining $\mathrm{BM}$ progenitors were incubated for 2 days in 6-well culture plates $\left(5 \times 10^{6}\right.$ cells/well) in DMEM medium supplemented with $10 \%$ FBS, recombinant murine-granulocyte macrophage colony-stimulating factor (GM-CSF; 1,000 IU/ml) and recombinant murine-interleukin-4 (IL-4; 1,000 IU/ml; both from PeproTech China) at $37^{\circ} \mathrm{C}$ in a $5 \% \mathrm{CO}_{2}$ incubator. After $48 \mathrm{~h}$, nonadherent granulocytes were gently removed and fresh medium was added. A total of $50 \%$ of the culture medium was replaced with fresh medium containing GM-CSF and IL-4 every $48 \mathrm{~h}$. On day 8 , the non-adherent cells were considered as immature DCs.

The immature DCs $\left(2 \times 10^{6}\right.$ cells $\left./ \mathrm{ml}\right)$ were washed twice with $3 \mathrm{ml}$ PBS and subsequently infected with either rAd-FAP- $\alpha$ or rAd-c, at a multiplicity of infection (MOI) of 300. Cells were incubated in 24 -well $\left(5 \times 10^{5}\right.$ cells $\left./ \mathrm{ml}\right)$ plates in $0.5 \mathrm{ml}$ of serum-free media supplemented with 1,000 IU/ml GM-CSF and $500 \mathrm{IU} / \mathrm{ml} \mathrm{IL-4}$ for $2 \mathrm{~h}$. Subsequently, complete media was added and cells were incubated for an additional 2 days. The infected DCs were harvested and washed twice with PBS, prior to subsequent experimentation. Since the recombinant 
adenoviral vector included GFP (Fig. 1A; plasmid map), transduction efficiency of DCs was determined by assessing GFP expression via flow cytometry analysis. Cell viability was assessed using trypan blue dye-exclusion (Sigma Aldrich; Merck KGaA). Briefly, the cell suspension was thoroughly mixed with $0.4 \%$ trypan blue solution at a ratio of $1: 1$. The number of viable and dead cells were counted using a hemocytometer (Thermo Fisher Scientific, Inc.), and the cell viability percentage was determined.

Flow cytometric analysis. On day 10 , rAd-FAP- $\alpha$ DCs, non-infected DCs or rAd-c DCs $\left(10^{6}\right.$ cells $\left./ \mathrm{ml}\right)$ were collected and resuspended in cold FACS buffer (eBioscience; Thermo Fisher Scientific, Inc.). A total of $100 \mu 1$ DCs were immunostained with antibodies against CD80 (cat. no. 12-0801-81), CD86 (cat. no. 12-0869-42) or MHC II (I-A/I-E; cat. no. 12-5321-81), and isotype-matched antibodies; $\operatorname{IgG}$ Isotype Control (cat. no. 12-4888-81), IgG2b kappa Isotype Control (cat. no. 12-4732-81) and IgG2b kappa Isotype Control (cat. no. 12-4031-80) in the dark for $30 \mathrm{~min}$ at $4^{\circ} \mathrm{C}$ (all 1:20 and from eBioscience; Thermo Fisher Scientific, Inc.). The DCs were subsequently resuspended in PBS and their phenotypes were analyzed using a flow cytometer (BD Biosciences).

Western blotting. Total protein was extracted from rAd-FAP- $\alpha$ DCs, rAd-c DCs, LLC cells or CAFs using RIPA buffer [150 mM NaCl, $100 \mathrm{mM}$ Tris ( $\mathrm{pH} 8.0$ ), $1 \%$ Triton X-100, $1 \%$ deoxycholic acid, $0.1 \%$ SDS, $5 \mathrm{mM}$ EDTA, $10 \mathrm{mM}$ $\mathrm{NaF}, 1 \mathrm{mM}$ sodium vanadate, $2 \mathrm{mM}$ leupeptin, $2 \mathrm{mM}$ aprotinin, $1 \mathrm{mM}$ phenylmethylsulfonyl fluoride and $1 \mathrm{mM}$ dithiothreitol] (eBioscience; Thermo Fisher Scientific, Inc.) on ice for $30 \mathrm{~min}$. Protein concentration was determined using the BCA Protein Assay kit (Thermo Fisher Scientific, Inc.). Proteins $(20 \mu \mathrm{g})$ were separated by $6 \%$ SDS-PAGE and transferred onto Hybond-polyvinylidene difluoride membranes. Membranes were blocked with 5\% non-fat milk in PBS at room temperature for $1 \mathrm{~h}$ and subsequently incubated with primary antibodies against FAP- $\alpha(1: 1,000$; cat. no. NB110-85534; Novus Biologicals, Ltd.) and GAPDH (1:1,000; cat. no. NB100-56875; Novus Biologicals, Ltd.) for $2 \mathrm{~h}$ at room temperature. Membranes were washed three times in TBST (5 min/wash) and further incubated with an alkaline phosphatase-conjugated goat anti-rabbit IgG secondary antibody (1:2,500; cat. no. ADI-SAB-301-J; Enzo Life Sciences Inc.) for $1 \mathrm{~h}$ at room temperature. Protein bands were visualized using the ECL western blot analysis system (GE Healthcare).

In vivo immunization of mice and tumor challenge study. Female C57BL/6 mice (H-2 $\left.{ }^{b}\right)$ were used in the in vivo experiments. In the prophylactic study, mice were initially vaccinated and then injected with tumor cells. Mice were divided into three groups ( $\mathrm{n}=10 \mathrm{mice} / \mathrm{group})$ and were immunized subcutaneously (s.c.) with rAd-FAP- $\alpha$ DCs, rAd-c DCs or DCs alone $\left(5 \times 10^{5}\right.$ cells/mouse), three times into the left flanks at 6 day intervals. Following the last immunization, after 1 week, $5 \times 10^{5}$ LLC cells were injected s.c. into the right flanks of the mice and the tumor of mice were observed for 8 weeks.

In the therapeutic efficacy study, mice were initially injected with tumor cells and then vaccinated. A total of $5 \times 10^{5} \mathrm{LLC}$ cells were s.c. injected into the right flank of each mouse. Mice began treatment when the tumor diameter reached 4-6 mm (at day 7). The mice were divided into three groups ( $\mathrm{n}=10$ mice/group) and injected s.c. with rAd-FAP- $\alpha$ DCs, rAd-c DCs or DCs alone $\left(5 \times 10^{5}\right.$ cells/mouse), three times into the left flanks at 4 day intervals. The shortest diameter (width) and the longest diameter (length) of each tumor were measured using a digital caliper every 2 days to determine the antitumor effect. Tumor volume was calculated as follows: $\mathrm{V}=$ length $\mathrm{x}$ width ${ }^{2} \mathrm{x} 0.52$. All mice were sacrificed according to the Institutional Animal Care and Use Committee protocol when they became moribund or when the tumor diameter reached $20 \mathrm{~mm}$, which were recorded as the date of death in the survival studies. The overall survival rates of the mice were assessed using SPSS 20.0 software (IBM Corp.).

CAFs cultures and immunofluorescence staining. LLC CAFs were obtained from the s.c. implanted LLC tumor by collagenase treatment, following separation with meshing by a nylon filter and adhesion of plastics, as previously described $(1,2)$. CAFs were incubated in DMEM media supplemented with $20 \% \mathrm{FBS}$ at $37^{\circ} \mathrm{C}$ and passaged every 3-4 days. After 5 passages, cells were fixed with $4 \%$ paraformaldehyde (BioSharp Life Sciences) for $15 \mathrm{~min}$, and subsequently permeabilized with $0.2 \%$ Triton-X-100 (Beijing Dingguo Changsheng Biotechnology Co., Ltd.) for $15 \mathrm{~min}$ at room temperature. Cell were blocked with $10 \%$ goat serum (Biological Industries) at room temperature for $1 \mathrm{~h}$ and incubated with the following primary antibodies: Rabbit anti- $\alpha$-smooth muscle actin (1:500; cat. no. NBP1-30894; Novus Biological, Ltd.), anti-FAP- $\alpha$ (1:500; cat. no. MAB9727; Novus Biological, Ltd.), anti-vimentin (1:500; cat. no. NBP1-97670; Novus Biological) or anti-pan cytokeratin (1:200; cat. no. NB600-579; Novus Biological) overnight at $4^{\circ} \mathrm{C}$. Cells were washed three times with PBS and subsequently incubated with a goat anti-rabbit IgG secondary antibody (1:500; cat no. SA00001-2; ProteinTech Group, Inc.) for $1 \mathrm{~h}$ at $37^{\circ} \mathrm{C}$. The nuclei of the CAFs were stained with DAPI (ProteinTech Group, Inc.) and cell images were captured using an OLYMPUS IX73 fluorescence microscope (magnification x 400; Olympus Corporation).

Cytotoxic T lymphocyte (CTL) assay. A CytoTox $96^{\circledR}$ non-radioactive cytotoxicity assay (Promega Corporation) was performed, according to the manufacturer's protocol to assess cytotoxic activity. Briefly, C57BL/6 mice (n=9; 3/group) were immunized s.c. with rAd-FAP- $\alpha$ DCs, rAd-c DCs or DCs alone $\left(5 \times 10^{5}\right.$ cells/mouse), two times at 6 day intervals to evaluate whether rAd-FAP- $\alpha$ DCs could induce antitumor specific CTLs. After the last immunization on day 6 mice were sacrificed. Splenocytes were obtained by gentle disruption of the spleen of the mice and filtration using a Falcon ${ }^{\circledR} 40 \mu \mathrm{m}$ Nylon cell strainer (Corning, Inc.). Subsequently, the splenocytes were treated with $\mathrm{RBC}$ lysis solution and further cultured at $37^{\circ} \mathrm{C}$ in complete medium for $90 \mathrm{~min}$. Nonadherent splenocytes $\left(3 \times 10^{6}\right.$ cells $\left./ \mathrm{ml}\right)$ were harvested and re-stimulated in 24-well culture plates using mitomycin C (Sigma-Aldrich; Merck KGaA)-treated CAFs $\left(10^{6}\right.$ cells $\left./ \mathrm{ml}\right)$, in the presence of recombinant murine IL-2 (10 U/ml; PeproTech). These splenocytes [effector cells (E)] were incubated at $37^{\circ} \mathrm{C}$ for 5 days. After 5 days, E were cultured with CAFs [target cells (T)] in round-bottom 96-well culture plates according to the E:T ratios 10:1, 30:1 and 50:1. After $4 \mathrm{~h}$, the plate was centrifuged at $250 \mathrm{xg}$ for $5 \mathrm{~min}$ at $4^{\circ} \mathrm{C}$ 

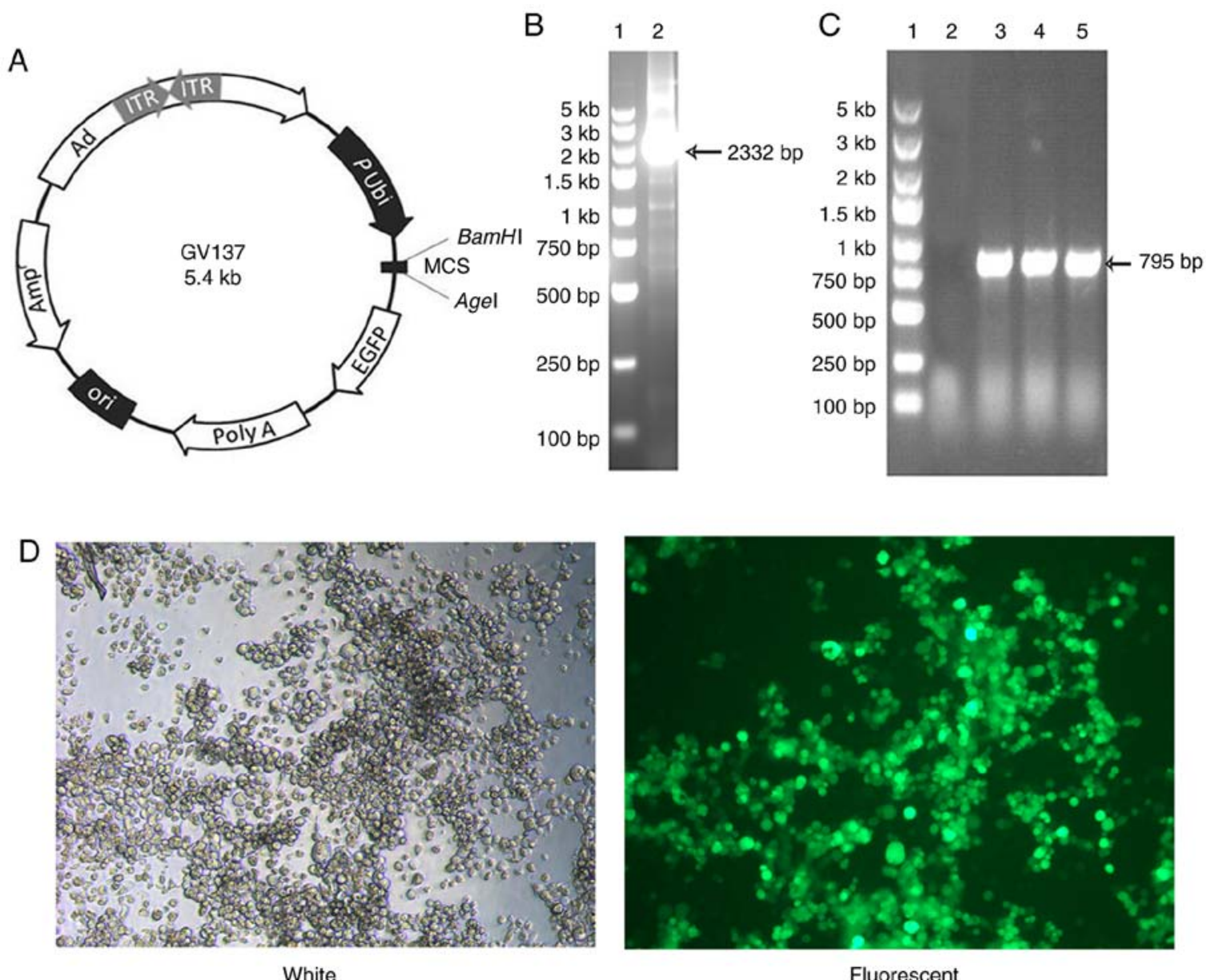

Fluorescent

Figure 1. Identification and construction of adenoviral vector encoding mouse FAP- $\alpha$. (A) GV137 plasmid map. (B) Identification of the amplified FAP- $\alpha$ circular DNA fragment. Lane 1, DNA marker (100 bp-5 kb ladder); lane 2, amplified FAP- $\alpha$ PCR products. (C) Identification of the recombinant GV137-FAP- $\alpha$ plasmid. Lane 1, DNA marker (100 bp-5 kb ladder); lane 2, negative control (empty vector control); lane 3-5, PCR products of the recombinant GV137-FAP- $\alpha$ plasmid. (D) GFP expression was detected via fluorescence microscopy (magnification x100). FAP- $\alpha$, fibroblast activation protein- $\alpha$; bp, base pair; kb, kilobase; Ad, adenovirus; ITR, inverted terminal repeats; $P$ Ubi, promoter from ubiquitin gene; MCS, multiple cloning site; EGFP, enhanced green fluorescent protein; Ori, origin of replication; Amp, ampicillin.

and the supernatants were collected. Lactate dehydrogenase maximal release was determined according to completely lysed $\mathrm{T}$. T cultured without $\mathrm{E}$ were considered for spontaneous release and used as the negative controls. The percentage of cytotoxicity (specific lysis) was measured using the following formula: [(experimental release-spontaneous release)/(maximum release-spontaneous release)] x100.

Statistical analysis. Statistical analysis was performed using SPSS 20.0 software (IBM Corp.) and data are presented as the mean \pm standard deviation. ANOVA followed by Bonferroni's post-hoc test were used to compare differences between multiple groups and two groups. Survival analysis of mice was performed using the log-rank test, followed by Bonferroni's post-hoc test. $\mathrm{P}<0.05$ was considered to indicate a statistically significant difference.

\section{Results}

Construction of recombinant adenovirus carrying FAP- $\alpha$. The cDNA encoding mouse FAP- $\alpha$ was amplified using primers. The amplified DNA fragment corresponded to the expected size segment $(2,332$ bp; Fig. 1B). FAP- $\alpha$ cDNA fragments were inserted into the GV137 shuttle plasmid (Fig. 1A) to yield GV137-FAP- $\alpha$, which was verified via PCR analysis. PCR amplification produced the expected size of the band (795 bp), confirming therefore that the recombinant GV137-FAP- $\alpha$ plasmid contained the target segment (Fig. 1C). The 293T cells were co-transfected with the GV137-FAP- $\alpha$ shuttle plasmid and pBHGlox $(\delta) E 1,3$ Cre helper plasmid, including the adenovirus backbone. The CPE was assessed via fluorescence microscopy, which verified production of the recombinant adenovirus (rAd-FAP- $\alpha$ ) 10 days post-cotransfection. rAd-FAP- $\alpha$ was subsequently infected with $293 \mathrm{~T}$ cells, and GFP expression was detected 2-3 days post-infection via fluorescence microscopy (Fig. 1D). The purified rAd-FAP- $\alpha$ particle titer was $2.2 \times 10^{10} \mathrm{TCID}_{50} / \mathrm{ml}$.

In vitro recombinant adenoviral infection of DCs. On day 7 of DC culture, DCs were infected with rAd-FAP- $\alpha$, and the efficiency of infection was assessed. The positive rate of GFP detected via flow cytometry analysis was $\sim 74.1 \%$ (Fig. 2A). MOI 300 was used for gene transduction in the present study as cell viability was $>80 \%$. 
A
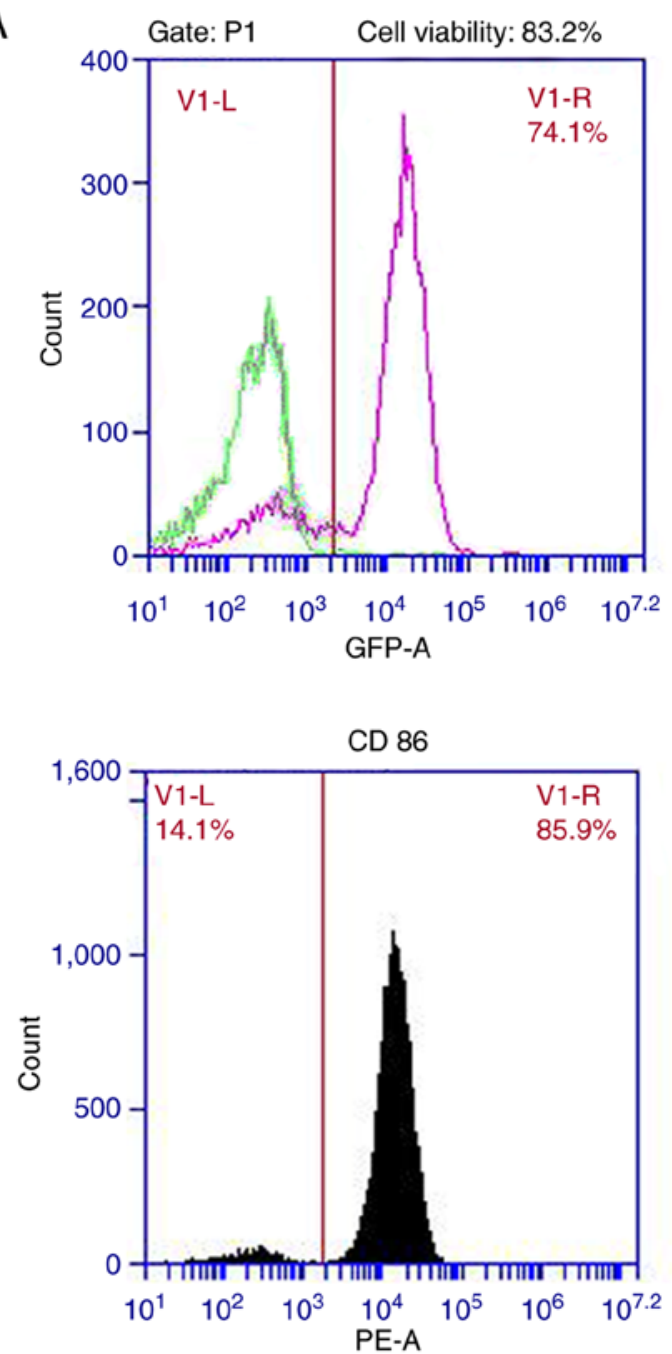

B
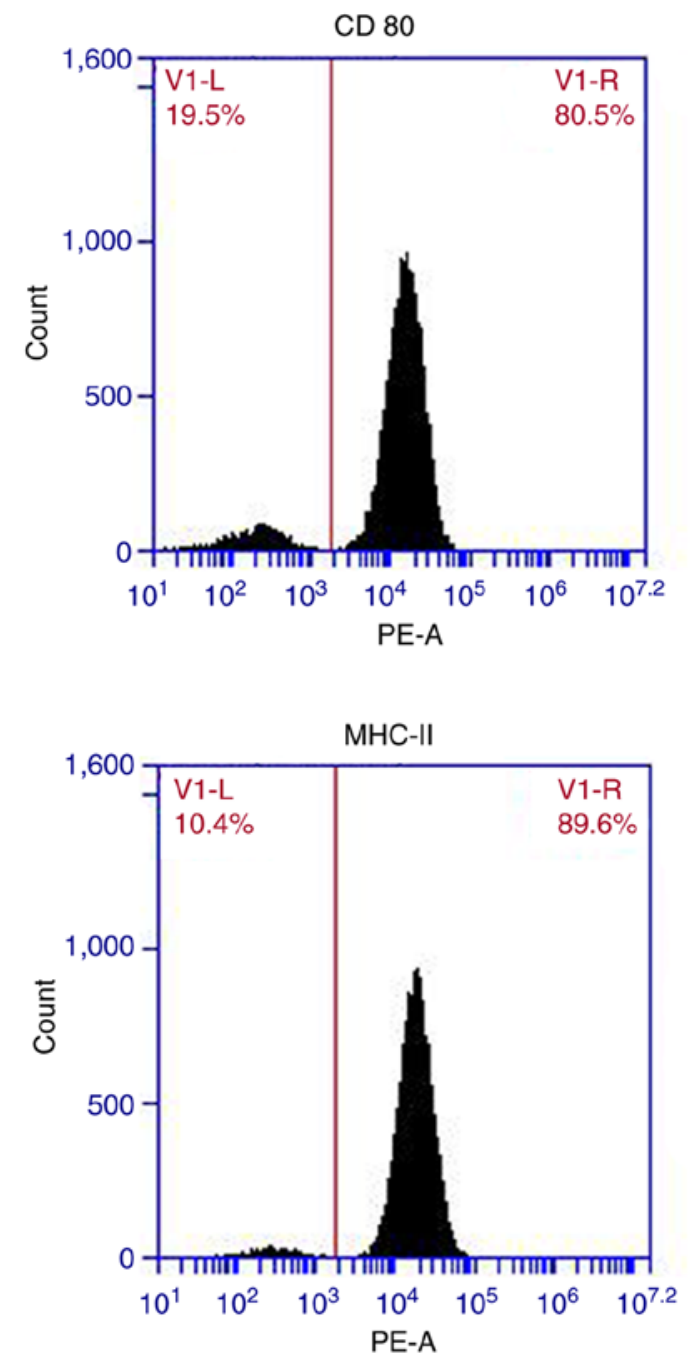

Figure 2. Efficiency of rAd-FAP- $\alpha$ infection and the phenotype of rAd-FAP- $\alpha$ DCs. (A) Cell viability of rAd-FAP- $\alpha$ DCs and efficacy of rAd-FAP- $\alpha$ transduction into DCs. GFP expression level was determined via flow cytometric analysis 2 days post-infection, and the percent rate of viable cells was assessed using the trypan blue exclusion test (V1-R, \% of GFP-positive cells). (B) Influence of rAd-FAP- $\alpha$ on phenotypes of DCs. Mice DCs were infected with either rAd-FAP- $\alpha$ or rAd-c at a multiplicity of infection of 300 and the expression levels of cell surface markers were assessed via flow cytometric analysis 2 days post-recombinant adenovirus infection. Eexpression levelsof CD80, CD86 and MHC class II in rAd-FAP- $\alpha$-infected DCs was 80.5, 85.9 and 89.6\%, respectively. rAd-FAP- $\alpha$ DCs, recombinant adenovirus-fibroblast activation protein- $\alpha$ dendritic cells; GFP, green fluorescent protein.

Effect of rAd-FAP- $\alpha$ infection on phenotype of DCs. The present study assessed the DC surface markers, MHC class II, CD86 and CD80, using flow cytometric analysis. The results demonstrated that the expression levels of CD80, CD86 and MHC class II in rAd-FAP- $\alpha$-infected DCs were $80.5,85.9$ and $89.6 \%$, respectively (Fig. 2B). No significant differences in the expression of the surface markers were observed between rAd-FAP- $\alpha$-infected DCs and rAd-c-infected DCs (data not shown).

CAFs cultures. CAFs were obtained from s.c. implanted LLC tumors by collagenase digestion and passaged every 4 days (Fig. 3A). After 4 passages, CAFs nuclei was stained using DAPI, $>95 \%$ of CAFs positively expressed vimentin (Fig. 3B), $\alpha$-SMA (Fig. 3C) and FAP- $\alpha$ (Fig. 3D) as demonstrated via immunostaining analysis.

FAP- $\alpha$ expression in CAFs and rAd-FA- $\alpha$ DCs. Previous studies have reported that FAP- $\alpha$ is expressed in CAFs but not in cancer cells $(22,23)$. In order to determine whether FAP- $\alpha$ displays a similar expression pattern in the experimental systems of the present study, FAP- $\alpha$ expression was assessed in a series of CAFs, LLC cells, rAd-c DCs and rAd-FAP- $\alpha$ DCs. The results demonstrated that mouse FAP- $\alpha$ expression was overexpressed in rAd-FAP- $\alpha$ DCs and CAFs; however, no expression was detected in rAd-c DCs and LLC cells (Fig. 3E). This suggests that rAd-FAP- $\alpha$ DCs vaccine had been successfully constructed, the CAFs used in present study were appropriate to be targeted for immunotherapy and LLC cells cannot be targeted by CTL induced by rAd-FAP- $\alpha$ DCs vaccine in subsequent experiments due to lack of FAP- $\alpha$ expression.

Tumor-specific CTL activity. The effector cells from C57BL/6 mice immunized with rAd-FAP- $\alpha$ DCs exhibited significantly cytotoxic effects in CTLs against the targets cells (CAFs) at E:T ratios of 30:1 and 50:1; however, the control effector cells from C57BL/6 mice immunized with non-transduced DCs or rAd-c DCs exhibited minimal 


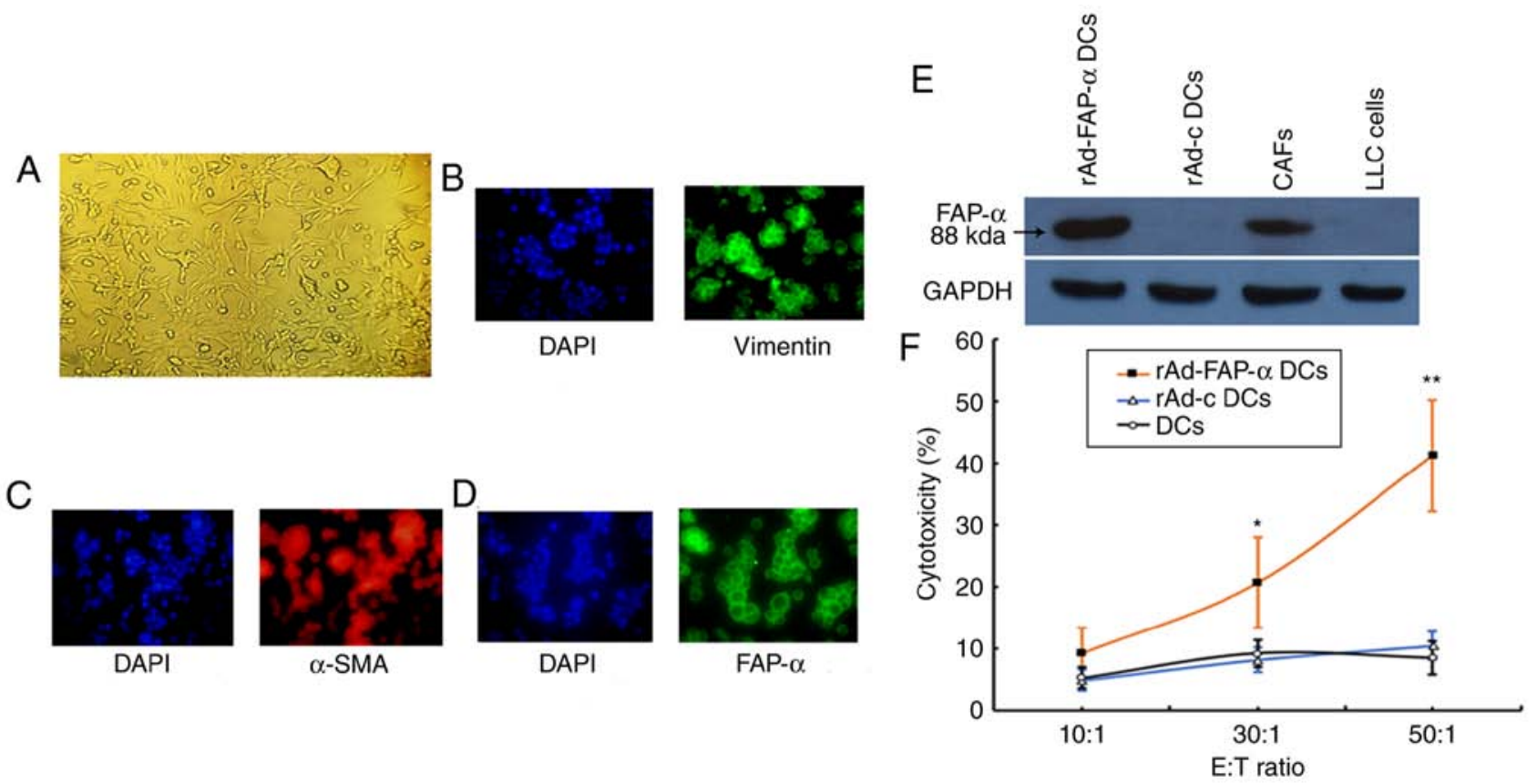

Figure 3. Identification of CAFs, western blot analysis of FAP- $\alpha$ expression and induction of CTL by immunizing mice with rAd-FAP- $\alpha$ DCs. (A) CAFs (magnification x200), DAPI staining of CAFs nuclei and (B) vimentin, (C) $\alpha$-SMA and (D) FAP- $\alpha$ immunofluorescence staining of CAFs (magnification x400). (E) Western blot analysis of FAP- $\alpha$ expression. LLC cells and rAd-c DCs were used as the negative controls, while GAPDH was used as the standard internal control. (F) Induction of tumor-specific cytotoxic T lymphocyte activity, following vaccination of mice with rAd-FAP- $\alpha$ DCs. ${ }^{*} \mathrm{P}<0.01$, ${ }^{* *} \mathrm{P}<0.001$ vs. rAd-c DCs group and DCs group. CAFs, cancer-associated associated fibroblasts; FAP- $\alpha$, fibroblast activation protein- $\alpha$; DCs, dendritic cells; $\alpha$-SMA, $\alpha$-smooth muscle actin.

cytotoxicity (Fig. 3F). Taken together, these results indicated that rAd-FAP- $\alpha$ DCs may have the ability to induce the production of specific CTLs against FAP- $\alpha$-positive CAFs.

Antitumor efficacy of $r A d-F A P-\alpha D C s$. In order to determine whether rAd-FAP- $\alpha$ DCs exhibit an antitumor function in vivo, the present study used LLC bearing mouse models [LLC $\left(\mathrm{H}-2^{\mathrm{b}}\right)$ in C57BL/6 mice $\left(\mathrm{H}-2^{\mathrm{b}}\right)$ ]. The results demonstrated that vaccination with rAd-FAP- $\alpha$ DCs induced significant antitumor effects and led to $100 \%$ mice survival compared with the rAd-c DCs and DCs groups (Fig. 4A).

Considering the results from the prophylactic study, an in vivo therapeutic experiment was performed to determine the therapeutic effects of vaccination with rAd-FAP- $\alpha$ DCs. The results demonstrated that vaccination with rAd-FAP- $\alpha$ DCs significantly inhibited tumor growth compared with the rAd-c DCs or DCs groups, during the observation period of 23 days, following s.c. injection with LLC cells (days 13-23, all $\mathrm{P}<0.001$; Fig. 4B). Furthermore, the tumor volumes in the rAd-FAP- $\alpha$ DCs group were significantly decreased compared with the rAd-c DCs and DCs groups at days 13-23 (Fig. 4C). The representative tumor volume image is from day 23 (Fig. 4D). However, no significant difference was observed in the tumor volume between the rAd-c DCs group and the DCs group. A total of 4/10 mice immunized with rAd-FAP- $\alpha$ DCs survived until day 61 of the observation period, while all mice in the other two groups either died of natural causes or were euthanized $(\mathrm{P}<0.001$; Fig. $4 \mathrm{C})$. Taken together, the results of the present study suggested that immunization with rAd-FAP- $\alpha$ DCs may inhibit tumor growth and increase the survival rates of LLC-bearing mice.
However, this effect failed to completely eradicate the tumor in the present study.

\section{Discussion}

The discovery that human melanomas could express a type of non-mutated tumor-associated antigen, which can spontaneously induce $\mathrm{CD} 8^{+} \mathrm{T}$ cell responses was a major advancement in the study of tumor immunology (24). However, therapeutic vaccines using such antigens lack efficiency in controlling tumor growth (25-27). Previous studies reported that tumors may induce immunological tolerance (25) or lose expression of the tumor cell surface antigen upon tumor progression $(26,27)$; however, these hypotheses fail to explain the occurrence of systemic tumor immune responses in patients with tumors that have been immunized using such antigens, alongside the fact that these immune responses cannot maintain or induce tumor regression, although the tumor continues to express tumor antigens and MHC class I $(28,29)$. These findings indicate that immunosuppression in the tumor microenvironment could be a major reason for the poor efficacy of therapeutic vaccination. The tumor microenvironment includes numerous types of stromal cells. Tumor cells are embedded in the tumor stroma, which is the connective tissue framework of several types of solid tumors. Thus, tumoral stroma cells may be a major determinant in the immune suppression of the tumor microenvironment $(6,30)$. Certain mesenchymal-derived stromal cells, including peritumoral and intratumoral CAFs, may be identified according to the expression of the type II membrane glycoprotein with intrinsic dipeptidyl-peptidase FAP- $\alpha$ (30), which is associated with immune suppression 
A

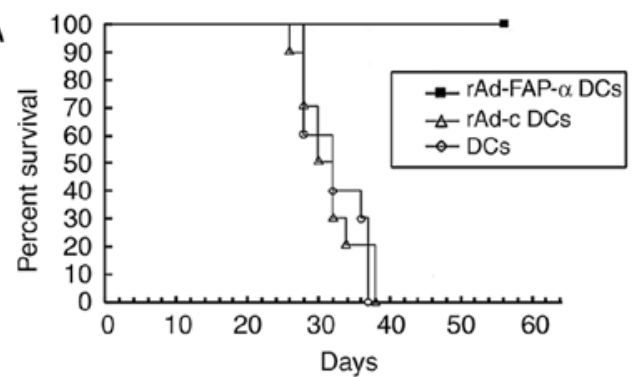

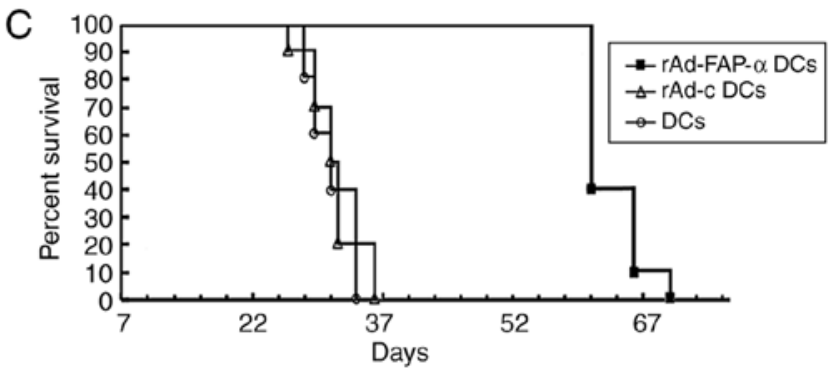

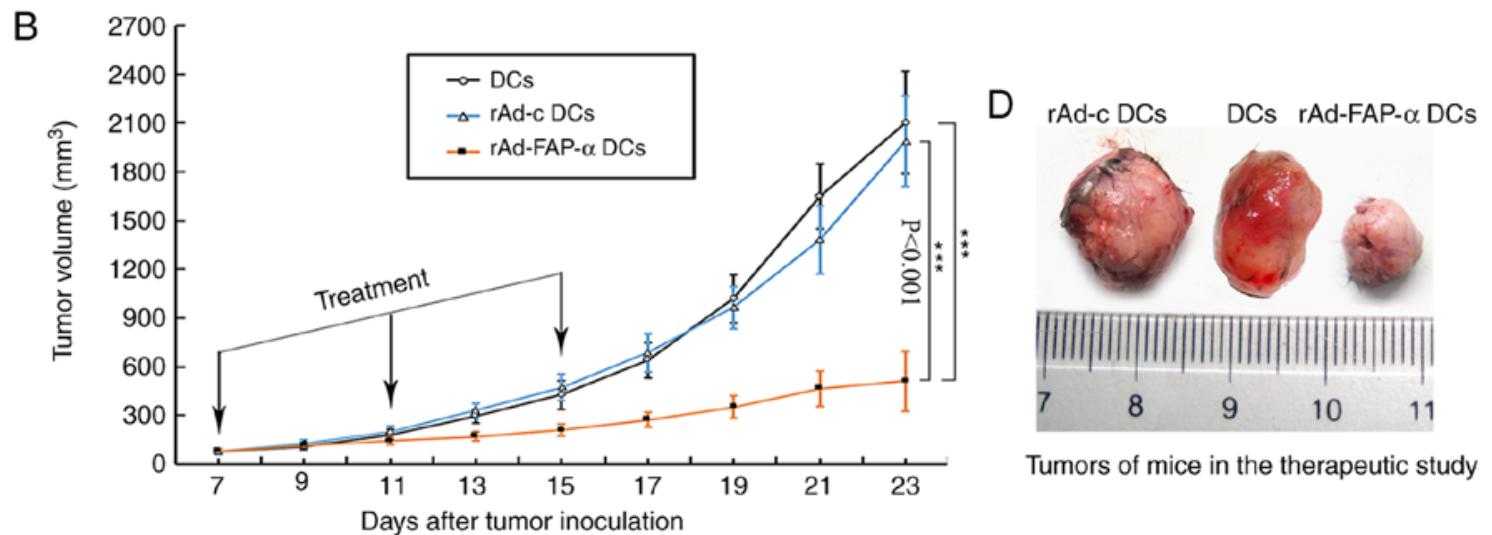

Figure 4. Efficacy of rAd-FAP- $\alpha$ DCs in antitumor progression and prolongation of mouse survival time. C57BL/6 mice were vaccinated s.c. with rAd-FAP- $\alpha$ DCs, rAd-c DCs or DCs. (A) In the prophylactic study, mice were injected s.c. with $5 \times 10^{5}$ LLC cells 1 week after the last vaccination. Mice were subsequently divided into three groups and overall survival time was assessed for 8 weeks. (B) In the therapeutic efficacy study, LLC cells $\left(5 x 10^{5}\right)$ were initially injected s.c. into the right flank of mice on the first day. After 7 days, mice were subsequently divided into three groups, according to tumor size. Tumor volumes were measured every 2 days, while (C) mice survival rates were observed for 70 days. (D) Tumors of mice in the therapeutic study, following s.c. injection with LLC cells at day 23. ${ }^{* * *} \mathrm{P}<0.001$ vs. rAd-c DCs group and DCs group. rAd-FAP- $\alpha$ DCs, recombinant adenovirus-fibroblast activation protein- $\alpha$ dendritic cells; s.c., subcutaneously; LLC, Lewis lung cancer.

and tumor growth (31-33). FAP- $\alpha$ is a type II transmembrane protein that belongs to the serine integral membrane peptidases (SIMPs) family. SIMPs also contain the cell surface serine protease dipeptidyl peptidase IV (DPPIV, also known as CD26) and dipeptidyl peptidase IIX $(34,35)$. These peptidases are active on the cell surface and are specific and inducible for proline-containing peptides $(34,35)$. According to previous clinical trials where the enzymatic activity of FAP- $\alpha$ was targeted $(36,37)$, and previous studies demonstrating FAP- $\alpha$ specific upregulation in $>90 \%$ of pancreatic, colon, breast and lung cancers $(23,38)$, treatment through targeting FAP- $\alpha$ may be considered as an effective therapeutic method for patients with various types of tumor.

In order to assess the antitumor immune effect of targeting FAP- $\alpha$, the present study designed a recombinant adenovirus containing the mouse FAP- $\alpha$ with the aim of transducing DCs and immunizing C57BL/6 mice. The results of the present study demonstrated that CTLs produced in mice using the vaccine targeting FAP- $\alpha$ may recognize the mouse FAP- $\alpha$-derived epitope and induce a significant cytotoxic effect. In order to determine the antitumor immunotherapy efficacy of rAd-FAP- $\alpha$ DCs vaccine in vivo, the present study used LLC bearing mouse models. The results demonstrated that vaccination with rAd-FAP- $\alpha$ DCs markedly increased the antitumor protection effects (in the prophylactic immunotherapy experiment, $100 \%$ for LLC tumor) and markedly delayed the growth of the established LLC tumor. However, rAd-c DCs had no significant effects on the antitumor protection efficacy of mice. Previous studies have also demonstrated the use of anti-stromal immunotherapy by targeting FAP- $\alpha$ (39-41) and reported results similar to those from the present study.

In summary, the present study demonstrated that targeting a surface type II membrane glycoprotein of CAFs named FAP- $\alpha$, which is overexpressed specifically and selectively on CAFs, may inhibit tumor growth in a mouse LLC model. FAP- $\alpha$ may be considered as a potential target for killing or destroying CAFs within the tumor stromal microenvironment, and may be used to develop immunogenic tumor vaccines. However, successful application of FAP- $\alpha$-targeted immune therapy in patients with lung cancer will require the development of more efficient vaccination protocols, and assessment in other lung cancer models. Further investigation is also required to determine the underlying mechanism by which targeting the FAP- $\alpha$ could inhibit tumor growth in vivo, either by direct inhibition of the CAFs or by appendant damages, such as secretion of cytokines (leading to a local inflammatory response) and a decrease in infiltrative immunosuppressive cells in the tumor microenvironment.

\section{Acknowledgements}

Not applicable.

\section{Funding}

The present study was funded by the National Natural Science Foundation of China (grant nos. 81160294 and 81960425) and the key projects of Science and Technology Department of Jiangxi Province (grant no. 20181BBH80005). 


\section{Availability of data and materials}

The datasets used and/or analyzed during the present study are available from the corresponding author upon reasonable request.

\section{Authors' contributions}

JX designed the present study, analyzed and interpreted the data, and drafted the initial manuscript. SY, LP, HL, LN performed the experiments. $\mathrm{HX}$ and $\mathrm{XG}$ performed flow cytometric analysis and collated the relevant literature. MY and FD analyzed the data and revised the manuscript. All authors read and approved the final manuscript.

\section{Ethics approval and consent to participate}

All experimental protocols were approved by the Ethics Committee of The Second Affiliated Hospital of Nanchang University (Nanchang, China; approval no. NDEFYEC 175-2018), and mice were sacrificed according to the Institutional Animal Care and Use Committee protocol.

\section{Patient consent for publication}

Not applicable.

\section{Competing interests}

The authors declare that they have no competing interests.

\section{References}

1. Lai D, Ma L and Wang F: Fibroblast activation protein regulates tumor-associated fibroblasts and epithelial ovarian cancer cells. Int J Oncol 41: 541-550, 2012.

2. Giannoni E, Bianchini F, Calorini L and Chiarugi P: Cancer associated fibroblasts exploit reactive oxygen species through a proinflammatory signature leading to epithelial mesenchymal transition and stemness. Antioxid Redox Signal 14: 2361-2371, 2011.

3. Puré E and Lo A: Can targeting stroma pave the way to enhanced antitumor immunity and immunotherapy of solid tumors? Cancer Immunol Res 4: 269-278, 2016.

4. Liu T, Zhou L, Li D, Andl T and Zhang Y: Cancer-associated fibroblasts build and secure the tumor microenvironment. Front Cell Dev Biol 7: 60, 2019.

5. Erez N, Truitt M, Olson P, Arron ST and Hanahan D: Cancer-associated fibroblasts are activated in incipient neoplasia to orchestrate tumor-promoting inflammation in an NF-kappaB-dependent manner. Cancer Cell 17: 135-147, 2010.

6. Ohshio Y, Teramoto K, Hanaoka J, Tezuka N, Itoh Y, Asai T, Daigo $\mathrm{Y}$ and Ogasawara K: Cancer-associated fibroblast-targeted strategy enhances antitumor immune responses in dendritic cell-based vaccine. Cancer Sci 106: 134-142, 2015.

7. Barron DA and Rowley DR: The reactive stroma microenvironment and prostate cancer progression. Endocr Relat Cancer 19: R187-R204, 2012.

8. Gascard P and Tlsty TD: Carcinoma-associated fibroblasts: Orchestrating the composition of malignancy. Genes Dev 30: 1002-1019, 2016.

9. Valkenburg KC, de Groot $\mathrm{AE}$ and Pienta KJ: Targeting the tumour stroma to improve cancer therapy. Nat Rev Clin Oncol 15: 366-381, 2018.

10. Elenbaas B and Weinberg RA: Heterotypic signaling between epithelial tumor cells and fibroblasts in carcinoma formation. Exp Cell Res 264: 169-184, 2001.

11. Xing F, Saidou J and Watabe K: Cancer associated fibroblasts (CAFs) in tumor microenvironment. Front Biosci (Landmark Ed) 15: 166-179, 2010 .
12. Bhowmick NA, Neilson EG and Moses HL: Stromal fibroblasts in cancer initiation and progression. Nature 432: 332-337, 2004.

13. Santos AM, Jung J, Aziz N, Kissil JL and Puré E: Targeting fibroblast activation protein inhibits tumor stromagenesis and growth in mice. J Clin Invest 119: 3613-3625, 2009.

14. Edosada CY, Quan C, Tran T, Pham V, Wiesmann C, Fairbrother W and Wolf BB: Peptide substrate profiling defines fibroblast activation protein as an endopeptidase of strict Gly(2)-Pro(1)-cleaving specificity. FEBS Lett 580: 1581-1586, 2006.

15. Edosada CY, Quan C, Wiesmann C, Tran T, Sutherlin D, Reynolds M, Elliott JM, Raab H, Fairbrother W and Wolf BB: Selective inhibition of fibroblast activation protein protease based on dipeptide substrate specificity. J Biol Chem 281: 7437-7444, 2006.

16. Jansen K, Heirbaut L, Cheng JD, Joossens J, Ryabtsova O, Cos P, Maes L, Lambeir AM, De Meester I, Augustyns K, et al: Selective inhibitors of fibroblast activation protein (FAP) with a (4-Quinolinoyl)-glycyl-2-cyanopyrrolidine scaffold. ACS Med Chem Lett 4: 491-496, 2013.

17. Meadows SA, Edosada CY, Mayeda M, Tran T, Quan C, Raab H, Wiesmann C and Wolf BB: Ala657 and conserved active site residues promote fibroblast activation protein endopeptidase activity via distinct mechanisms of transition state stabilization. Biochemistry 46: 4598-4605, 2007.

18. Ostermann E, Garin-Chesa P, Heider KH, Kalat M, Lamche H, Puri C, Kerjaschki D, Rettig WJ and Adolf GR: Effective immunoconjugate therapy in cancer models targeting a serine protease of tumor fibroblasts. Clin Cancer Res 14: 4584-4592, 2008.

19. Jia J, Martin TA, Ye L and Jiang WG: FAP- $\alpha$ (Fibroblast activation protein- $\alpha$ ) is involved in the control of human breast cancer cell line growth and motility via the FAK pathway. BMC Cell Biol 15: 16, 2014.

20. Nyberg-Hoffman C, Shabram P, Li W, Giroux D and Aguilar-Cordova E: Sensitivity and reproducibility in adenoviral infectious titer determination. Nat Med 3: 808-811, 1997.

21. Xie J, Xiong L, Tao X, Li X, Su Y, Hou X and Shi H: Antitumor effects of murine bone marrow-derived dendritic cells infected with xenogeneic livin alpha recombinant adenoviral vectors against lewis lung carcinoma. Lung Cancer 68: 338-345, 2010.

22. Lee J, Fassnacht M, Nair S, Boczkowski D and Gilboa E: Tumor immunotherapy targeting fibroblast activation protein, a product expressed in tumor-associated fibroblasts. Cancer Res 65: 11156-11163, 2005.

23. Puré E and Blomberg R: Pro-tumorigenic roles of fibroblast activation protein in cancer: Back to the basics. Oncogene 37: 4343-4357, 2018.

24. van der Bruggen $\mathrm{P}$, Traversari $\mathrm{C}$, Chomez $\mathrm{P}$, Lurquin $\mathrm{C}$, De Plaen E, Van den Eynde B, Knuth A and Boon T: A gene encoding an antigen recognized by cytolytic $\mathrm{T}$ lymphocytes on a human melanoma. Science 254: 1643-1647, 1991.

25. Willimsky G, Czéh M, Loddenkemper C, Gellermann J, Schmidt K, Wust P, Stein H and Blankenstein T: Immunogenicity of premalignant lesions is the primary cause of general cytotoxic T lymphocyte unresponsiveness. J Exp Med 205: 1687-1700, 2008.

26. Dunn GP, Koebel CM and Schreiber RD: Interferons, immunity and cancer immunoediting. Nat Rev Immunol 6: 836-848, 2006.

27. Speiser DE, Baumgaertner P, Barbey C, Rubio-Godoy V, Moulin A, Corthesy P, Devevre E, Dietrich PY, Rimoldi D, Liénard D, et al: A novel approach to characterize clonality and differentiation of human melanoma-specific $\mathrm{T}$ cell responses: Spontaneous priming and efficient boosting by vaccination. J Immunol 177: 1338-1348, 2006.

28. Rosenberg SA, Sherry RM, Morton KE, Scharfman WJ, Yang JC, Topalian SL, Royal RE, Kammula U, Restifo NP, Hughes MS, et al: Tumor progression can occur despite the induction of very high levels of self/tumor antigen-specific CD8 + T cells in patients with melanoma. J Immunol 175: 6169-6176, 2005.

29. Valmori D, Souleimanian NE, Tosello V, Bhardwaj N, Adams S, O'Neill D, Pavlick A, Escalon JB, Cruz CM, Angiulli A, et al: Vaccination with NY-ESO-1 protein and CpG in montanide induces integrated antibody/Th1 responses and CD8 T cells through cross-priming. Proc Natl Acad Sci USA 104: 8947-8952, 2007.

30. Garin-Chesa P, Old LJ and Rettig WJ: Cell surface glycoprotein of reactive stromal fibroblasts as a potential antibody target in human epithelial cancers. Proc Natl Acad Sci USA 87: 7235-7239, 1990. 
31. Dolznig H, Schweifer N, Puri C, Kraut N, Rettig WJ, Kerjaschki D and Garin-Chesa P: Characterization of cancer stroma markers: In silico analysis of an mRNA expression database for fibroblast activation protein and endosialin. Cancer Immun 5: 10, 2005

32. Scarfò I and Maus MV: Current approaches to increase CAR T cell potency in solid tumors: Targeting the tumor microenvironment. J Immunother Cancer 5: 28, 2017.

33. Orimo A,Gupta PB, SgroiDC, Arenzana-Seisdedos F, Delaunay T, Naeem R, Carey VJ, Richardson AL and Weinberg RA: Stromal fibroblasts present in invasive human breast carcinomas promote tumor growth and angiogenesis through elevated SDF-1/CXCL12 secretion. Cell 121: 335-348, 2005.

34. Chen WT and Kelly T: Seprase complexes in cellular invasiveness. Cancer Metastasis Rev 22: 259-269, 2003.

35. Rosenblum JS and Kozarich JW: Prolyl peptidases: A serine protease subfamily with high potential for drug discovery. Curr Opin Chem Biol 7: 496-504, 2003.

36. Hofheinz RD, al-Batran SE, Hartmann F, Hartung G, Jäger D, Renner C, Tanswell P, Kunz U, Amelsberg A, Kuthan H and Stehle G: Stromal antigen targeting by a humanised monoclonal antibody: An early phase II trial of sibrotuzumab in patients with metastatic colorectal cancer. Onkologie 26: 44-48, 2003.

37. Scott AM, Wiseman G, Welt S, Adjei A, Lee FT, Hopkins W, Divgi CR, Hanson LH, Mitchell P, Gansen DN, et al: A phase dose-escalation study of sibrotuzumab in patients with advanced or metastatic fibroblast activation protein-positive cancer. Clin Cancer Res 9: 1639-1647, 2003.
38. Kawase T, Yasui Y, Nishina S, Hara Y, Yanatori I, Tomiyama Y, Nakashima Y, Yoshida K, Kishi F, Nakamura M and Hino K: Fibroblast activation protein- $\alpha$-expressing fibroblasts promote the progression of pancreatic ductal adenocarcinoma. BMC Gastroenterol 15: 109, 2015.

39. Brennen WN, Rosen DM, Wang H, Isaacs JT and Denmeade SR: Targeting carcinoma-associated fibroblasts within the tumor stroma with a fibroblast activation protein-activated prodrug. J Natl Cancer Inst 104: 1320-1334, 2012.

40. Schreiber H and Rowley DA: Cancer. Awakening immunity. Science 330: 761-762, 2010

41. Kraman M, Bambrough PJ, Arnold JN, Roberts EW, Magiera L, Jones JO, Gopinathan A, Tuveson DA and Fearon DT: Suppression of antitumor immunity by stromal cells expressing fibroblast activation protein-alpha. Science 330: 827-830, 2010.

(i) $(5)$ This work is licensed under a Creative Commons Attribution-NonCommercial-NoDerivatives 4.0 International (CC BY-NC-ND 4.0) License. 